\title{
Atuaçáo do Terapeuta Ocupacional com criança com atraso do desenvolvimento neuropsicomotor
}

\author{
Ariana Carramaschi de Souza ${ }^{a}$, Milena de Souza Fazio Marino ${ }^{a}$ \\ aTerapeuta ocupacional, Hospital das Clínicas, Faculdade de Medicina de Ribeirão Preto - FMRP, \\ Universidade de São Paulo - USP, Ribeirão Preto, SP, Brasil
}

\begin{abstract}
Resumo: A prematuridade refere-se ao nascimento de bebês com idade gestacional inferior a 37 semanas. Sabe-se que essa condição, associada a uma internação prolongada, constitui um fator de risco ao desenvolvimento neuropsicomotor. Este estudo teve por objetivo ressaltar os ganhos referentes aos componentes de desempenho sensoriais, neuromusculoesqueléticos e motores ocorridos após as intervenções em terapia ocupacional com uma criança pré-termo. Com este propósito foi feito levantamento bibliográfico e atendimentos semanais de Terapia Ocupacional no Centro de Reabilitação da Rede Lucy Montoro do Hospital das Clínicas da Faculdade de Medicina de Ribeirão Preto, onde se utilizou a escala de avaliação Alberta Infant Motor Scale (AIMS) em uma criança prematura extrema, a fim de avaliar e quantificar seu desempenho motor. Após a avaliação, foram utilizados recursos lúdicos com o propósito de estimular os componentes de desempenho sensoriais, neuromusculoesqueléticos e motores para que, posteriormente, os mesmos auxiliem em uma maior independência durante a participação efetiva no brincar. Verificou-se que a intervenção realizada proporcionou o desenvolvimento de um repertório de ações sensoriais, neuromusculoesqueléticas e motoras, processo por meio do qual, ao se fazer a correção da idade cronológica pelo fator da prematuridade, observou-se que os ganhos foram extremamente satisfatórios e condizentes com o esperado para a idade corrigida, evidenciando a efetividade da atuação do terapeuta ocupacional como agente minimizador dos efeitos causados pelos fatores de risco ao desenvolvimento neuropsicomotor.
\end{abstract}

Palavras-chave: Prematuridade, Terapia Ocupacional, Desenvolvimento Motor, AIMS.

\section{The role of the Occupational Therapist with children with neuropsychomotor development delay}

\begin{abstract}
Prematurity it is the birth of infants before 37 weeks gestation; it is known that such a condition associated with prolonged hospitalization is a risk factor to the development of infants. This study aimed to highlight the gains related to sensorial, neuromuscleskeletal and motor performance components occurred after occupational therapy intervention in a preterm infant. To this end, a literature survey as carried out, as well as weekly assistance of occupational therapy at the Lucy Montoro rehabilitation center in Ribeirao Preto, state of Sao Paulo, where the Alberta Infant Motor Scale (AIMS) was used in an extremely preterm infant in order to evaluate and quantify motor performance. After assessment, we used playful resources to improve sensorial neuromuscleskeletal motor performance components so that, later, they assist in greater independence for effective participation in the playing. We verified that the intervention brought about the development of a repertoire of sensorial neuromuscleskeletal motor actions; through this process, when correction of the chronological age was made by the prematurity factor, it was possible to observe that the gains were very satisfactory and consistent with what is expected for the corrected age, demonstrating the effectiveness of occupational therapy assistance as an agent for minimizing the effects caused by risk factors to neuropsychomotor development.
\end{abstract}

Keywords: Prematurity, Occupational Therapy, Motor Development, AIMS.

Autor para correspondência: Ariana Carramaschi de Souza, Hospital das Clínicas, Faculdade de Medicina de Ribeirão Preto, Universidade de São Paulo, Av. Bandeirantes, 3900, Centro de Reabilitação, Monte Alegre, CEP 14048-900, Ribeirão Preto, SP, Brasil,

e-mail: nanacarramaschi@hotmail.com

Recebido em 5/11/2010; $1^{\text {a }}$ Revisão em 24/10/2011; $2^{\text {a }}$ Revisão em 6/2/2012; $3^{\text {a }}$ Revisão em 29/5/2012; Aceito em 11/6/2012. 


\section{Introdução}

A Organização Mundial da Saúde (OMS) define como prematuro o recém-nascido $(\mathrm{RN})$ com menos de 37 semanas de gestação, independente do peso ao nascimento. As crianças nascidas com menos de 2.500 gramas sáo classificadas como recém-nascidos de baixo peso, não importando o tempo de gestação (MARQUES, 2003).

Estudos sobre esses neonatos demonstram que a sobrevivência nos quadros mais severos de prematuridade vem aumentando, graças aos avanços tecnológicos no cuidado intensivo neonatal (CARVALHO; MAGALHÃES, 2004).

Estima-se que $25 \%$ dos bebês que requerem esse tipo de assistência são considerados de risco, sujeitos a comprometimento neurológico ou retardo no desenvolvimento (UMPHRED, 2003).

A literatura aponta que os critérios indicadores de risco, tais como a prematuridade, o baixo peso e a anóxia perinatal, são vistos como importantes determinantes de anormalidades no desenvolvimento neurossensóriomotor. Apesar de esses fatores atentarem para um déficit neurológico, existe uma dificuldade em detectar essas anormalidades nos primeiros quatro meses, pois esse período é caracterizado pelo comportamento variável do bebê, bem como por alteraçôes do tônus muscular, atividades postural e reflexa e habilidades funcionais (SOUZA, 1998).

Segundo sua idade gestacional, os prematuros podem ser classificados como: prematuro limítrofe, entre 37 e 38 semanas; prematuro moderado, entre 31 e 36 semanas; e prematuro extremo, entre 24 e 30 semanas, sendo que esses apresentam maior instabilidade clínica (RIOS, 2007).

Independente da classificação, o prematuro tem como característica uma imaturidade global, nascendo em condiçôes clínicas instáveis, com variado grau de dificuldades anatômicas e funcionais. Hospitalizado por tempo indefinido, ele acaba por continuar seu desenvolvimento sob condiçôes não fisiológicas, gerando riscos para seu sistema nervoso central (SNC), com possível acometimento de sequelas (HERNANDEZ, 2003).

Dentre as sequelas que atingem os bebês prematuros, a curto prazo destacam-se a doença da membrana de hialina, a retinopatia da prematuridade, a hemorragia peri-intraventricular, a leucomalacia periventricular, a enterocolite necrosante, sepse e displasia broncopulmonar. Já nos prematuros extremos, o que mais se observa a longo prazo é a paralisia cerebral, com uma incidência de aproximadamente dois recém-nascidos para cada mil nascidos vivos (SILVEIRA; PROCIANOY, 2005).

Nesse grupo de neonatos é comum a ocorrência de distúrbios neuromotores entre os sobreviventes, fato que tem estimulado o surgimento de programas para acompanhamento dessas crianças, que são consideradas de risco para atraso no desenvolvimento neuropsicomotor (MANCINE et al., 2002).

Sabendo que essas condiçóes adversas no nascimento podem gerar problemas a curto, médio e longo prazos, afetando assim o desenvolvimento da criança, é importante intervir para neutralizar ou minimizar os efeitos cumulativos dos riscos (PEDREMÔNICO, 2003).

A intervenção no paciente pré-termo deve ser global e multidisciplinar, atentando não só para a necessidade diagnóstica como para as necessidades terapêuticas, visando assim a integralidade da criança. Assim, além das ações básicas, como medicamentos, alimentação e higiene, são estabelecidas outras intervençôes como o brincar e o vínculo mãe e filho (FURTADO; LIMA, 1999).

Soares e Zamberlan (2001) consideram o brincar como uma atividade que pode facilitar o trabalho dos profissionais de saúde porque propicia melhor adaptação e cooperação por parte da criança, além de auxiliar no seu desenvolvimento, incrementando seu repertório comportamental.

Considerando-se o brincar como o fazer e o papel ocupacional da criança, observa-se no terapeuta ocupacional, como estudioso do fazer humano, um profissional capacitado para analisar e avaliar o brincar, podendo planejar intervençóes e obter informaçóes sobre as competências cognitivas, motoras e sociais da criança (KNOX, 2002).

A Terapia Ocupacional busca favorecer o desempenho ocupacional da criança, focando o desenvolvimento necessário das habilidades adequadas a sua faixa etária, proporcionando açóes através da brincadeira, cuja importância se verifica não somente por ser vista como meio para atingir a melhora dos componentes de desempenho, mas porque a brincadeira é entendida, também, como atividade lúdica, integrando a área de desenvolvimento do brincar (GRIGOLATTO et al., 2008).

Levando-se em consideração o levantamento bibliográfico aqui exposto, a prática da Terapia Ocupacional foi desenvolvida com o intuito de favorecer o desenvolvimento de uma criança prematura extrema, considerando-se os principais marcos do desenvolvimento infantil e a divisão em áreas, componentes e contextos de desempenho. 
A publicaçáo deste relato foi autorizada pelo Comitê de Ética do Hospital das Clínicas da Faculdade de Medicina de Ribeirão Preto. A exibição das imagens publicadas também foi autorizada pelos responsáveis por elas.

\section{Objetivo}

O objetivo deste trabalho é relatar a experiência de uma intervenção clínica realizada em uma criança prematura extrema, no centro de reabilitação da Rede Lucy Montoro do Hospital das Clínicas da Faculdade de Medicina de Ribeirão Preto, no intuito de ressaltar os ganhos relacionados aos componentes de desempenho sensoriais (tato, visão, audição, percepção e equilíbrio), neuromusculoesqueléticos (força, resistência e controle postural) e motores (integração bilateral, integração visomotora e controlemotor) ocorridos após as intervençôes da Terapia Ocupacional, tendo-se utilizado como recurso, nessas intervençôes, atividades lúdicas.

\section{Metodologia}

Para o presente estudo foram realizadas intervençôes terapêuticas no período de fevereiro a junho de 2010 em uma criança do sexo feminino, com diagnóstico de prematuridade extrema, idade gestacional de 25 semanas e 4 dias, pesando $640 \mathrm{~g}$, apgar 1 e 3 nos primeiros cinco minutos e 1 no $10^{\circ}$ minuto, com 2 anos e 2 meses de idade, que foi submetida a atendimentos semanais e individuais em Terapia Ocupacional, na Rede Lucy Montoro do Hospital das Clínicas da Faculdade de Medicina de Ribeirão Preto.

Foram feitos levantamentos bibliográficos através de livros texto e das bases de dados Scielo, Cadernos de Terapia Ocupacional da Universidade Federal de São Carlos e Revista de Terapia Ocupacional da Universidade de São Paulo, a fim de aprofundar o conhecimento sobre prematuridade e auxiliar na atuação prática.

Para a coleta de dados relacionada ao quadro clínico da criança, reuniram-se informaçóes do prontuário, que possui registro de cirurgias, exames, procedimentos, avaliaçóes e atendimentos realizados por toda a equipe multiprofissional de saúde.

A avaliação da Terapia Ocupacional foi feita considerando-se os principais marcos do desenvolvimento infantil e a divisão em áreas, componentes e contextos de desempenho proposta pela American Occupational Therapy Association - AOTA
(AMERICAN..., 2002). Durante a avaliação foram observados o movimento ativo e o posicionamento da criança no tablado, bem como sua resposta (sensorial, neuromusculoesquelética e motora) aos estímulos lúdicos apresentados. Também foi feita a aplicação da escala padronizada de avaliação Alberta Infant Motor Scale (AIMS), que é uma escala observacional construída para medir a maturação motora ampla em bebês, desde o nascimento até a locomoção independente (PIPER; DARRAH, 1994). Tal avaliação, validada e padronizada para uma amostra de crianças na província de Alberta, no Canadá, foi criada com o objetivo de avaliar o desenvolvimento motor dos recém-nascidos a termo e dos pré-termos de 0 a 18 meses de idade. É uma medida que aborda conceitos do desenvolvimento motor tais como neuromaturaçáo, perspectivas do sistema dinâmico e avaliação da sequência do desenvolvimento motor. Os padróes motores e posturas são analisados com base em três critérios: alinhamento postural, movimentos antigravitacionais e superfície de contato.

A avaliação inclui 58 itens, organizados em quatro posiçóes (supina, prona, sentada e de pé) e dispostos da seguinte maneira: $21 \mathrm{em}$ prona, $9 \mathrm{em}$ supina, 12 na posiçáo sentada e 16 em pé. Para cada item, certos descritores chave são identificados e observados para que o bebê seja ou não aprovado nesse determinado item. Cada item descreve três aspectos do desempenho motor: sustentação do peso, postura e movimentos antigravitacionais. A pontuação é anotada como observada ou não observada e, assim, para cada item observado o examinador atribui um ponto e, para cada item não observado, zero ponto (ALMEIDA et al., 2008). Ao final, os pontos em cada postura são somados em uma pontuação total de itens observados.

Os objetivos foram traçados comparando-se os dados obtidos na escala AIMS de avaliação inicial e final da criança, utilizando-se como estratégias e recursos de intervenção brinquedos que propiciassem o estímulo de cada componente de desempenho desejado, como também o interesse demonstrado pela criança durante as sessóes.

\section{Resultados e discussão}

A avaliação inicial de Terapia Ocupacional utilizando-se o AIMS foi realizada quando a criança estava com 1 ano e 9 meses. Observou-se que a criança realizava de forma satisfatória 50 dos 58 itens distribuídos entre as posturas da seguinte forma: 19 itens em prono, 8 itens em supino, 10 itens sentada e 13 itens em pé. Após a avaliação, 
considerando-se os itens observados e não observados e os aspectos do desempenho motor relacionados a eles (sustentaçáo de peso, postura e movimentos antigravitacionais), assim como a idade corrigida devido ao fator prematuridade, constatou-se o atraso no desenvolvimento neuropsicomotor.

Foram, entáo, iniciados os atendimentos de Terapia Ocupacional no Centro de Reabilitação da Rede Lucy Montoro do Hospital das Clínicas da Faculdade de Medicina de Ribeirão Preto, de forma individual, uma vez por semana e com duraçáo de 1 hora, num total de 19 atendimentos no período de fevereiro a junho de 2010.

De acordo com os déficits motores observados após a aplicação do AIMS e seguindo a classificação proposta pela AOTA (AMERICAN..., 2002), a intervenção da Terapia Ocupacional teve como foco o estímulo dos seguintes componentes de desempenho: sensoriais (tato, visão, audição, propriocepção e vestibular); neuromusculoesqueléticos (força, resistência e controle postural); e motores (integração bilateral, integração visomotora e controlemotor).

Todos os componentes foram estimulados de forma lúdica. Assim, para estimular os componentes sensoriais foram oferecidos à criança brinquedos e objetos com diferentes texturas, cores, contrastes e luz; quanto aos estímulos dos componentes neuromusculoesqueléticos e motores, foram proporcionadas trocas posturais (de supino para prono, de supino para decúbitos laterais direito e esquerdo, de decúbitos laterais para sentar e de sentada para ajoelhada, semiajoelhada e em pé), sempre utilizando brinquedos coloridos e com sons, a fim de atrair a atenção da criança e tornar o posicionamento e a aquisição das trocas um momento prazeroso. Observou-se que após esses atendimentos a criança passou a realizar essas trocas espontaneamente e a iniciar as brincadeiras sempre na posição ortostática. Foram realizadas atividades sobre superfícies instáveis, como a bola suíça, a fim de proporcionar maior propriocepção, estimular o equilíbrio e a reação de proteção. Estimulou-se descarga de peso em membros superiores e membros inferiores e a marcha foi iniciada através da atividade de faz de conta com "carrinho de boneca".

A mãe, presente durante os atendimentos, assumiu papel de facilitadora do processo terapêutico. Ela foi orientada a estimular a criança quanto aos aspectos sensoriais (oferecer diferentes texturas durante o banho e as brincadeiras, usar brinquedos coloridos e sonoros) e motores (permanência na postura ortostática durante as atividades em casa), no dia a dia ou nos dias em que a criança não participasse do atendimento de Terapia Ocupacional.
A avaliação final, utilizando-se a AIMS, foi realizada quando a criança estava com 2 anos e 2 meses. Observou-se que além dos 50 itens que já realizava anteriormente de forma satisfatória, ela passou a realizar 57 itens, distribuídos entre as posturas: 21 itens em prono, $9 \mathrm{em}$ supino, 12 sentada e 15 em pé. $O$ único item náo observado foi a postura em pé durante a marcha com total independência.

Comparando-se as avaliaçóes inicial e final fica evidente que a intervenção do terapeuta ocupacional, como agente, foi de extrema importância no auxílio à criança durante o seu processo de desenvolvimento, uma vez que, a partir dos estímulos oferecidos e das trocas posturais vivenciadas, ela desenvolveu um repertório de açóes que se encontravam atrasadas, as quais, futuramente, poderão transformar-se em esquemas de açôes mais elaboradas.

\section{Conclusão}

A partir do levantamento bibliográfico foi possível refletir sobre como os fatores de risco podem afetar o desempenho ocupacional da criança e compreender a relevância que, diante desse quadro, a Terapia Ocupacional pode ter para os pacientes.

Nota-se o quanto a atuação do terapeuta ocupacional pode ser benéfica e significativa, no sentido de maximizar o desempenho ocupacional satisfatório, contribuindo assim para uma melhora na qualidade de vida.

A estimulação descrita nesse relato foi feita sempre de forma lúdica, utilizando-se o brincar como estratégia de intervenção nos componentes de desempenho a serem alcançados pela criança. O brincar se caracterizar como uma das principais áreas de desempenho da infância.

Por meio da estimulação dos componentes de desempenho foi possível proporcionar alguma independência à criança na realização de suas atividades de vida diária, na participaçáo efetiva no brincar e no lazer.

Ainda existem objetivos a serem alcançados e a intervenção terapêutica deve continuar, porém muito já foi conquistado, demonstrando a importância da atuação do terapeuta ocupacional na intervençáo precoce.

\section{Referências}

ALMEIDA, K. M. et al. Validade concorrente e confiabilidade da Alberta Infant Motor Scale em lactentes nascidos prematuros. Jornal de Pediatria, Rio de Janeiro, v. 84 , n. 5 , p. 442-448, set./out. 2008. 
AMERICAN OCCUPATIONAL THERAPY ASSOCIATION - AOTA. Occupational Therapy Practice Framework: domain and process. American Journal of Occupational Therapy, New York, v. 56, n. 6, p. 609-633, Nov 2002. http://dx.doi.org/10.5014/ajot.56.6.609

CARVAlHO, D. J.; MAGALHĀES, L. C. A relação entre o desenho da figura humana e a coordenação visomotora em crianças pré-termo aos 6 anos de idade. Revista de Terapia Ocupacional da USP, São Paulo, v. 15, n. 3, p. 98-105, set./dez. 2004.

FURTADO, M. C. C.; LIMA, R. A. G. Brincar no Hospital: subsídios para o cuidado de enfermagem. Revista da Escola de Enfermagem da USP, São Paulo, v. 33, n. 4, p. 364-369, 1999. http://dx.doi.org/10.1590/ S0080-62341999000400007

GRIGOLATTO, T. et al. Intervenção Terapêutica Ocupacional em CTI Pediátrico: um estudo de caso. Cadernos de Terapia Ocupacional da UFSCar, São Carlos, v. 16, n. 1, p. 37-46, 2008.

HERNANDEZ, A. M. O Neonato. São José dos Campos: Pulso, 2003. (Coleção Cefac).

KNOX, S. Avaliação lúdica de pré-escolares: a escala Knox. In: PARHAM, L. D.; FAZIO, L. S. A recreação na terapia ocupacional pediátrica. São Paulo: Santos, 2002. p. 2-22.

MANCINI, M. C. et al. Estudo do desenvolvimento da função motora aos 8 e 12 meses de idade em crianças nascidas pré-termo e a termo. Arquivos de Neuro-Psiquiatria, São Paulo, v. 60, n. 4, p. 974-980, dez. 2002. http://dx.doi. org/10.1590/S0004-282X2002000600017
MARQUES, C. S. B. Classificação do recém-nascido segundo idade gestacional e crescimento fetal. In: PACHI, P. R. (Org.). O pré-termo: morbidade, diagnóstico e tratamento. Sáo Paulo: Roca, 2003. p. 23-40.

PEDREMÔNICO, M. R. M. Problemas de Desenvolvimento da Criança: prevenção e intervenção. In: ENCONTRO DE ESTUDOS DO DESENVOLVIMENTO HUMANO EM CONDIÇÓEES ESPECIAIS, 2., 2003, São Paulo. Temas sobre Desenvolvimento, São Paulo, v. 12, p. 7-9, 2003. Suplemento Especial.

PIPER, M. C.; DARRAH, J. Motor Assessment of the Developing Infant. Alberta: Saunders, 1994.

RIOS, I. J. Mãe e bebê prematuro extremo: possibilidade de vínculo em situação adversa. 2007. 85 f. Tese (Mestrado em Fonoaudiologia)-Pontifícia Universidade Católica de São Paulo, São Paulo, 2007.

SILVEIRA, R. C.; PROCIANOY, R. S. Lesôes isquêmicas cerebrais no recém-nascido pré-termo de muito baixo peso. Jornal de Pediatria, Rio de Janeiro, v. 81, n. 1, p. 23-32, 2005. Suplemento Especial.

SOARES, M. R. Z.; ZAMBERLAN, M. A. T. A Inclusão do Brincar na Hospitalização Infantil. Estudos de Psicologia, Campinas, v. 18, n. 2, p. 64-69, maio/ago. 2001.

SOUZA, R. C. T. Vigilância neuromotora de lactentes acometidos por indicadores de risco para asfixia perinatal no primeiro trimestre de vida. 1998. $95 \mathrm{f}$. Tese (Mestrado em Neurociências)-Universidade Estadual de Campinas, Campinas, 1998.

UMPHRED, D. A. Reabilitação Neurológica. São Paulo: Manole, 2003.

\section{Contribuição dos Autores}

Ariana Carramaschi de Souza: Concepção do texto, organização de fontes, redação do texto e revisão. Milena de Souza Fazio Marino: Revisão do texto. 